\title{
The efficacy of controlled release D-sotalol-polyurethane epicardial implants for ventricular arrhythmias due to acute ischemia in dogs
}

\author{
Vinod Labhasetwar, Alan Kadish, Thomas Underwood, Matthew Sirinek and Robert \\ J. Levy \\ Department of Pediatrics, University of Michigan Medical School, Ann Arbor, Michigan, USA
}

(Received 22 April 1992; accepted in revised form 9 September 1992)

Epicardially implanted D-sotalol polyurethane composite matrices for preventing ischemic ventricular arrhythmias were studied in open chest dogs under general anesthesia. D-sotalol was combined with a polyureapolyurethane (3:7) in solvent-cast films, which were characterized in vitro for their drug release at $37^{\circ} \mathrm{C}$ at $\mathrm{pH} 7.4\left(0.05 \mathrm{M} \mathrm{K}_{2} \mathrm{HPO}_{4}\right)$. D-sotalol in vitro release occurred rapidly in an initial burst phase, with roughly $20 \%$ released within the first five min, and $90 \%$ by $60 \mathrm{~min}$. Thereafter, an exponentially decreasing release rate was observed with matrix depletion by five hours. In the animal studies, the left anterior descending coronary artery (LAD) was occluded for 10 min on an hourly basis for up to five occlusions. 10 min prior to the third LAD occlusion, either a D-sotalol matrix or a vehicle matrix (control) was placed on either the ischemic or nonischemic left ventricular epicardium. The study was then continued observing the effects of matrix placement on occlusions 3, 4, and 5. 200 mg D-sotalol matrices, which delivered a net dose of $1.2 \mathrm{mg} / \mathrm{kg}$, effectively inhibited ventricular arrhythmias only if placed on the left ventricular ischemic zone. Placement of $200 \mathrm{mg}$ D-sotalol matrices in the nonischemic zone was ineffective for significantly reducing the occurrence of ventricular arrhythmias. Furthermore, D-sotalol controlled release matrices were ineffective for preventing ventricular fibrillation (VF) regardless of dose or placement site. $200 \mathrm{mg}$ ischemic zone $\mathrm{D}$-sotalol matrices resulted in plasma sotalol levels in regional coronary venous samples ranging from $3.5 \mu \mathrm{g} / \mathrm{ml}$ to 10.4 $\mu \mathrm{g} / \mathrm{ml}$. However, peripheral sotalol levels obtained simultaneously ranged from $0.23 \mu \mathrm{g} / \mathrm{ml}$ to $0.78 \mu \mathrm{g} /$ ml. It is concluded that epicardial D-sotalol controlled release matrices inhibited ischemic ventricular arrhythmias, but not VF, if placed in the left ventricular ischemic zone during repeated LAD occlusions.

Key words: Sustained release preparations; Drug delivery; Sotalol; Ventricular arrhythmias

\section{Introduction}

Therapy for ventricular arrhythmias is limited

Correspondence to: R.J. Levy, M.D. Kresge II, Room 5014, University of Michigan Ann Arbor, MI 48109-0576, U.S.A. Tel.: (313) 936-2850 Fax: (313) 764-4279. by a variety of factors including cardiac and noncardiac side effects. Previous research from our laboratory has demonstrated that drug-polymer composites, known as controlled release matrices, when placed on the epicardial left ventricular surface in open chest dogs, were effective for preventing ventricular arrhythmias in a variety 
of animal models [1-6]. For example, dogs with ouabain induced ventricular tachycardia were successfully converted to sinus rhythm with lidocaine-polyurethane controlled release matrices placed on the left ventricular epicardium [13]. Furthermore, ventricular tachycardia induced by rapid ventricular pacing was also successfully converted to sinus rhythm in an open chest dog model with placement of lidocaine-polyurethane matrices on the left ventricular epicardium $[4,5]$.

Ischemia-induced ventricular tachycardia in dogs during hourly $10 \mathrm{~min}$, complete occlusions of the left anterior descending coronary artery (LAD) was similarly significantly inhibited due to the placement of verapamil-polyurethane controlled release matrices on either the ischemic or nonischemic regions of the left ventricular epicardium [6]. In addition, related experiments also demonstrated that epicardial antiarrhythmic drug matrices were not associated with any drug-related cardiac or systemic side effects $[5,6]$.

Recent clinical and laboratory research has emphasized the importance of Class III antiarrhythmia agents for the therapy of ventricular arrhythmias. Sotalol is an interesting compound in this regard, since the l-stereoisomer has both beta-adrenergic blocking effects and Class III antiarrhythmic activity [7,8], whereas the D-stereoisomer has been reported to have predominantly Class III antiarrhythmic activity and is almost devoid of $\beta$-blocking effects $[9,10]$. For this reason, D-sotalol was chosen for use as a model Class III compound in these further investigations of controlled release implants for ventricular arrhythmias due to acute coronary ischemia. The goals of the present study were to: (1) formulate and characterize D-sotalol-polyurethane controlled release matrices in terms of their in vitro drug release; (2) assess the efficacy of D-sotalol controlled release matrices for inhibiting ischemia-induced ventricular arrhythmias occurring as a result of repeated acute LAD occlusions in a canine model; and (3) determine the myocardial and plasma disposition of $\mathrm{D}$-sotalol administration by epicardial matrices.

\section{Methods}

\section{Materials}

D-sotalol- $\mathrm{HCl}$ (MJ-5763-1-D) and the internal standard (MJ-6564-1) were obtained from Bristol Meyers Squibb, Inc. (Wallingford, CT). Methane sulfonic acid, $N, N$-dimethyl acetamide and 1-heptane sulfonic acid were purchased from Aldrich Chemical Company (Milwaukee, WI), and $N, N$-bis (2-hydroxyethyl)-glycine and procainamide hydrochloride were obtained from Sigma Chemical, Inc. (St. Louis, MO). Chloroform (Mallinckrodt, St. Louis, MO), methanol (Mallinckrodt) and isoamyl alcohol (J.D. Baker, Phillipsburg, NJ) were all high performance liquid chromatography grade. Acetic acid, sulfuric acid, sodium hydroxide, sodium chloride, potassium phosphate-dibasic, and potassium phosphate monobasic were all retained at reagent grade purity (Mallinckrodt, Paris, KY).

\section{Controlled release matrix formulation and characterization}

Mitralthane MPU5 (Polymedica, Inc., Wheatridge, $\mathrm{CO}$ ) was dissolved in dimethyl acetamide as a $25 \%$ solution. D-sotalol was codissolved in the dimethyl acetamide-MPU5 solution in proportions adjusted to give a final composition of 30\% D-sotalol and 70\% MPU5. Matrices were then solvent cast in teflon coated molds, and oven dried under vacuum at $50^{\circ} \mathrm{C}$. Solvent free matrices were 600 microns in thickness.

Individual slab matrices were incubated in 5 $\mathrm{ml}$ sample volumes of $0.05 \mathrm{M} \mathrm{K}_{2} \mathrm{HPO}_{4}(\mathrm{pH} 7.4$ ) at $37^{\circ} \mathrm{C}$ on an orbit shaker ( $110 \mathrm{rpm}$ ) under perfect sink conditions. The buffer was replenished on a regular basis and samples were periodically analyzed to monitor in vitro drug release. Sotalol concentration was determined either by absorbance at a wavelength of $227 \mathrm{~nm}$ using a Perkin Elmer Lambda 3B spectrophotometer (Norwalk, CT), or by high performance liquid chromatography (see below). 


\section{Animal model preparation}

Each experiment utilized an open-chest dog preparation [6] subjected to hourly complete occlusions of the left anterior descending coronary artery (LAD) for $10 \mathrm{~min}$ followed by reperfusion. The use of animals conformed to the "position of the American Heart Association on Research Animal Use", adopted Nov. 11, 1984. Five LAD occlusions were carried out with each animal with polyurethane matrix placement (drug or vehicle) prior to the third occlusion as described below. Thirty five male mongrel dogs (20 to $30 \mathrm{~kg}$ weight range) were used in these experiments. Animals were anesthetized with sodium pentobarbital, and the right femoral artery catheter was placed for monitoring blood pressure continuously during the studies. In addition, a right femoral venous catheter was also inserted for blood sampling. A left thoracotomy was then performed, and a pericardial cradle created. The left anterior descending coronary artery (LAD) was isolated and dissected proximal to its first branch. A snare consisting of a 2-0 silk ligature was placed around the LAD for periodic occlusions. Obvious left ventricular collateral branches communicating with the LAD distribution were tied off with 3-0 silk sutures. Unipolar epicardial platinum electrodes were placed in the ischemic zone and nonischemic zone of the left ventricle, and a bipolar epicardial platinum electrode was sewn in place onto the left atrial appendage for atrial pacing. In addition, all animals were configured with standard surface electrocardiogram electrodes and lead II was typically recorded. All data were continuously recorded on a Hewlett Packard Model PDR HP 8 channel FM open reel tape recorder (HewlettPackard, Andover, MA). Recorded data was also displayed on line using a Codas analogue to digital conversion system (DATA Q, Akron, $\mathrm{OH}$ ).

\section{LAD occlusion and drug protocols}

Two matrix sizes were used in the animal studies, either $200 \mathrm{mg}$ or $100 \mathrm{mg}$ net weight per matrix. Each D-sotalol-MPU5 matrix (30\% Dsotalol) or vehicle (control) matrix was sewn into place with epicardial sutures (3-0 vicryl) 10 min prior to the third LAD occlusion. Following this, the third occlusion was carried out on schedule, as well as fourth and fifth occlusion with a one hour interval between each occlusion. Once drug matrices were placed, heparinized blood samples were periodically removed from a coronary vein proximal to the drug matrix and simultaneously from the right femoral vein. At the conclusion of the study, animals were euthanized with an overdose of pentobarbital followed by injection of concentrated potassium chloride. After this, full thickness samples of myocardium from directly beneath the drug matrix, and $2 \mathrm{~cm}$ distal from it, were taken for subsequent sotalol analyses (see below). Furthermore, each D-sotalol matrix was stored frozen for subsequent analysis of residual drug.

Animals which failed to develop ventricular tachycardia during the first LAD occlusion were used for in vivo matrix release investigations. In these experiments, seven $10.0 \mathrm{mg}$ matrix samples were placed on the left ventricular epicardial surface along the LAD distribution distal to the occluder. Matrix samples were removed at intervals of $15,30,45,60,90,120$ and $130 \mathrm{~min}$ and were stored frozen for subsequent D-sotalol extraction and analysis by HPLC (see below).

\section{Ventricular arrhythmia analyses}

Animals were continuously paced during these studies (cycle length, $330 \mathrm{~ms}$ ) via the left atrial electrodes using a Grass Model 2000 Stimulator (Grass Instruments, Quincy, MA) set at twice diastolic capture thresholds. The following ventricular arrhythmias were noted during acute occlusions: isolated ventricular premature beats were recorded and quantitated, as were couplets. Episodes of nonsustained ventricular tachycardia, defined as three or more consecutive premature ventricular beats, were also noted and quantitated. Animals developing ventricular fibrillation (VF) during acute LAD occlusions, were assessed as to the duration of the occlusion until VF occurred. In addition, the occurrence of ventricular fibrillation during reperfusion was also noted. Animals with VF were promptly elec- 
trically defibrillated, and the study continued.

Ventricular arrhythmia data were compared between control and individual treated groups using unpaired t-test for each individual occlusion. This was necessary since repeated LAD occlusions lead to myocardial stunning [11] and other accumulative effects which thereby obviate comparing successive occlusions to each other within an experimental group. Ventricular fibrillation episodes, either during ischemia or during reperfusion, were similarly compared for each occlusion interval, control versus individual treatment group, for each of the various treatment regimes. Statistical significance was assessed for the occurrence of VF with chi square testing.

\section{D-Sotalol extraction procedures and HPLC analyses}

D-Sotalol in the plasma samples was quantified by HPLC using an ultraviolet detector, whereas myocardial samples were assayed using a fluorescence detector. MJ-6564-1 was used as an internal standard for plasma and matrix analyses and procainamide hydrochloride for myocardial samples. Stock solutions of D-sotalol hydrochloride and the internal standards were prepared in distilled water and were stored at $4^{\circ} \mathrm{C}$. The buffer solution was prepared from a saturated solution of N,N-bis (2-hydroxyethyl)glycine in distilled water, filtered and adjusted to pH 9.0 with $10 \mathrm{M}$ sodium hydroxide $[12,13]$.

Each $1 \mathrm{ml}$ plasma sample was first spiked with $100 \mu \mathrm{l}$ of internal standard and $200 \mu \mathrm{l}$ of buffer solution ( $\mathrm{pH} \mathrm{9.0),} \mathrm{and} \mathrm{then} \mathrm{extracted} \mathrm{with} 8 \mathrm{ml}$ extracting solvent consisting of a mixture of chloroform and isoamyl alcohol $(3: 1 \mathrm{v} / \mathrm{v})$. The organic layer $(5 \mathrm{ml})$ was removed through the lower phase and then mixed with $300 \mu$ of 0.05 $M$ sulfuric acid. An aliquot of the upper layer $(100 \mu \mathrm{l})$ was then injected into the reversedphase column.

Myocardial samples for sotalol analyses were first grossly stratified into epicardial, midmyocardial, and endocardial layers. Stratified samples $(0.5 \mathrm{~g})$ were homogenized with $2.2 \mathrm{ml} 0.9 \%$ $\mathrm{NaCl}$ and then treated in the same way as plasma samples except that procainamide hydrochloride was used as an internal standard. Matrix samples for assessment of residual D-sotalol content were extracted in refluxing methanol using a Soxhlet apparatus. Methanol extracts were evaporated to dryness and redissolved in $0.05 \mathrm{M}$ sulfuric acid for HPLC analyses using MJ-6564$l$ as an internal standard.

D-sotalol separations were carried out using a Waters (Milford, MA) HPLC system consisting of an HPLC pump (Model 501), a WISP 712 autosampler, a $30 \mathrm{~cm} \times 3.9 \mathrm{~mm}$ (internal diameter) reversed-phase $\mu$-Bondpack $\mathrm{C}_{18}(10 \mu$ particle size) with a RLSS Guard-pak precolumn insert with $10 \mu$ particles, a variable wavelength U.V. detector, Model 481 , set at $235 \mathrm{~nm}$., a scanning fluorescence detector, Model 470, set at excitation wavelength 235 and emission wavelength at $320 \mathrm{~nm}$, and a Data Module 740 recorder.

The mobile phase $[12,13]$ for plasma and matrix analyses was water-methanol-acetic acidmethanesulfonic acid (91:8.5:0.5:0.025 v/v). The mixture was adjusted to $\mathrm{pH} 3.3$ with $10 \mathrm{M}$ sodium hydroxide, filtered through a $0.45 \mu \mathrm{m}$ filter and vacuum degassed before use. The chromatography flow rate was $1.5 \mathrm{ml} / \mathrm{min}$, which produced a pressure $1500 \mathrm{psi}$. However, the mobile phase for myocardial sample analyses was methanol-acetic acid-water $(35: 1: 64 \mathrm{v} / \mathrm{v})$ with $0.005 \mathrm{M} \mathrm{w} / \mathrm{v}$ 1-heptane sulfonic acid [12-14]. It was also filtered through a $0.45 \mu \mathrm{m}$ filter and vacuum degassed before use. The HPLC flow rate was also $1.5 \mathrm{ml}$, which resulted in a pressure of 3300 psi.

All separations were made at ambient temperature, and peak areas were reported. Five working standard plasma (or tissue) samples were prepared from appropriate dilutions of stock solutions of D-sotalol hydrochloride to give concentrations ranging from 0 to $4000 \mathrm{ng} / \mathrm{ml}$ ( 0 to $10000 \mathrm{ng} / \mathrm{g}$ for myocardial tissue ). Calibration curves were constructed by plotting sotalol/internal standard peak area ratios from the extracted samples versus the sample sotalol concentration. The statistical significance of the differences between groups of plasma level sam- 
ples, or myocardial samples was assessed using unpaired $\mathrm{t}$-tests.

\section{Results}

D-Sotalol release in vitro occurred rapidly, with an initial burst phase of roughly $20 \%$ of incorporated drug within the first 5 min (Fig. 1). Furthermore, by $60 \mathrm{~min}$ about $90 \%$ of the cumulative release had occurred. Thereafter, an exponentially decreasing release rate was observed with nearly complete matrix depletion after 5 hours of in vitro release. Five dogs were excluded from the arrhythmia efficacy study

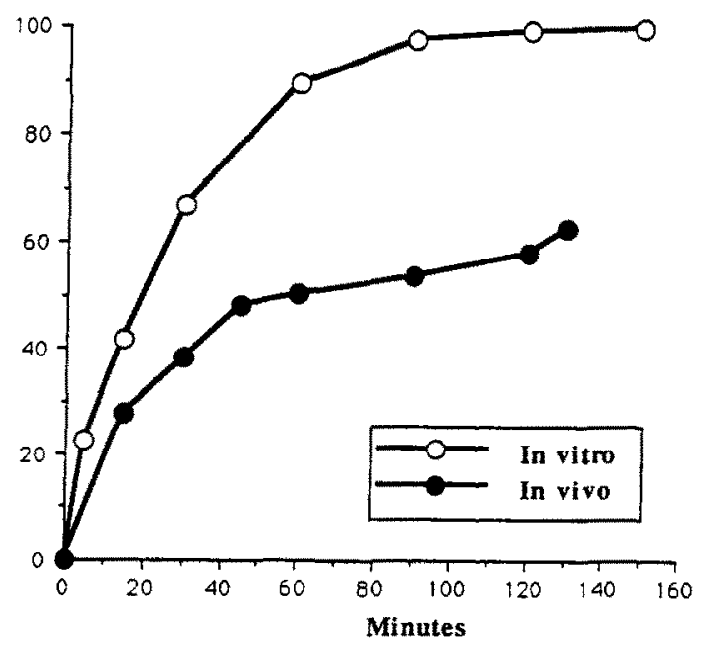

Fig. 1. In vitro (triplicate samples) compared to in vivo $(N=5)$ release of $\mathrm{D}$-sotalol from polyurethane matrices, shown as percentage of cumulative release. (below) because of the absence of VT during the first occlusion; however, these animals were utilized for in vivo matrix release experiments (as above). The in vivo sotalol release pattern was comparable to that observed in vitro, except that the release rate was roughly one half of that noted in vitro (Fig. 1).

Prior to matrix placement LAD occlusions resulted in multiform unstable ventricular ectopy with progressive severity during the course of each 10 min occlusion. Nonsustained VT typically occurred for 3 to 15 consecutive beats in control occlusions (prior to matrix placement, 1 and 2 ) and vehicle occlusions (3,4,5, nondrug matrices). In all studies in which nonsustained ventricular tachycardia (VT) was induced during the first occlusion, it recurred at a comparable frequency (Table 1 ), during the second occlusion, and for the remaining vehicle studies during occlusions 3-5 as well.

The occurrences of isolated premature beats and couplets are presented in Table 2 and Table 3. Couplets occurred at a rate of $1.24-2.29 / \mathrm{min}$ in both D-sotalol (pretreatment) and vehicle animals during the first occlusions (Table 2). In addition isolated premature beats also occurred frequently during nontreatment occlusions as well (Table 3). Ventricular fibrillation occurred after $5.1-7.3 \mathrm{~min}$ in $57 \%$ to $85 \%$ of animals in the various groups during the first occlusions (Table 4). Furthermore, ventricular fibrillation occurred during reflow in all untreated dogs not fibrillating during the LAD occlusion, and thus, undergoing reperfusion after $10 \mathrm{~min}$ of acute is-

TABLE I

Inhibition of isolated ventricular premature beats with $\mathrm{D}$-sotalol matrices (episodes/min)

\begin{tabular}{|c|c|c|c|c|c|c|}
\hline \multirow{2}{*}{$\begin{array}{l}\text { Group } \\
\text { (matrix wt) }\end{array}$} & \multirow[t]{2}{*}{$N$} & \multicolumn{5}{|l|}{ Occlusion } \\
\hline & & 1 & II & III & IV & V \\
\hline Control (vehicle) & 9 & $3.85 \pm 1.03$ & $3.01 \pm 0.65$ & $2.63 \pm 0.97$ & $2.75 \pm 0.98$ & $2.17 \pm 0.67$ \\
\hline $200 \mathrm{mg} \mathrm{IZ}$ & 7 & $5.07 \pm 1.50$ & $1.47 \pm 0.47$ & $1.18 \pm 0.39$ & $0.63 \pm 0.20^{*}$ & $0.37 \pm 0.17^{* *}$ \\
\hline $200 \mathrm{mg}$ NIZ & 7 & $3.81 \pm 2.11$ & $5.33 \pm 2.49$ & $1.59 \pm 0.48$ & $2.45 \pm 0.99$ & $0.74 \pm 0.43$ \\
\hline
\end{tabular}

$\mathrm{IZ}=$ ischemic zone matrix placement, 3rd occlusion; NIZ = nonischemic zone matrix placement, $3 \mathrm{rd}$ occlusion; ${ }^{*} p=0.051$, $w 1=2.11$, treated vs. control; ${ }^{* *} p=0.03, t=2.36$, treated vs. control. 
TABLE 2

Inhibition of couplets with D-sotalol matrices (episodes/min)

\begin{tabular}{lllllll}
\hline $\begin{array}{l}\text { Group } \\
\text { (matrix wt.) }\end{array}$ & $N$ & \multicolumn{2}{l}{ Occlusion* } & & \\
\cline { 2 - 6 } & & I & II & III & IV & V \\
\hline Control (vehicle) & 9 & $1.37 \pm 0.53$ & $0.51 \pm 0.17$ & $0.68 \pm 0.21$ & $0.47 \pm 0.15$ & $0.57 \pm 0.21$ \\
$200 \mathrm{mg}$ & 7 & $2.29 \pm 0.98$ & $0.41 \pm 0.13$ & $0.57 \pm 0.17$ & $0.14 \pm 0.09$ & $0.15 \pm 0.05$ \\
$200 \mathrm{mg} \mathrm{NIZ}$ & 7 & $1.24 \pm 0.68$ & $1.73 \pm 0.78$ & $0.61 \pm 0.20$ & $0.79 \pm 0.42$ & $0.32 \pm 0.30$ \\
$100 \mathrm{mg} \mathrm{IZ}$ & 7 & $1.37 \pm 0.14$ & $0.55 \pm 0.42$ & $0.48 \pm 0.19$ & $0.49 \pm 0.38$ & $0.50 \pm 0.14$ \\
\hline
\end{tabular}

$\mathrm{IZ}=$ ischemic zone matrix placement, 3rd occlusion; NIZ=nonischemic zone matrix placement, 3rd occlusion; * $p>0.05$ for control vs. treated for all occlusions.

\section{TABLE 3}

Inhibition of ventricular tachycardia with D-sotalol matrices (episodes/min)

\begin{tabular}{lllllll}
\hline $\begin{array}{l}\text { Group } \\
\text { (matrix wt.) }\end{array}$ & $N$ & \multicolumn{2}{l}{ Occlusion* } & & \\
\cline { 2 - 6 } & & I & II & III & IV & V \\
\hline Control (vehicle) & 9 & $1.25 \pm 0.27$ & $1.18 \pm 0.41$ & $0.74 \pm 0.22$ & $1.53 \pm 0.74$ & $1.02 \pm 0.36$ \\
$200 \mathrm{mg} \mathrm{IZ}$ & 7 & $1.48 \pm 0.44$ & $0.75 \pm 0.28$ & $0.84 \pm 0.16$ & $0.57 \pm 0.16$ & $0.33 \pm 0.125$ \\
$200 \mathrm{mg} \mathrm{NIZ}$ & 7 & $1.42 \pm 0.53$ & $1.50 \pm 0.60$ & $1.28 \pm 0.29$ & $1.28 \pm 0.52$ & $0.88 \pm 0.41$ \\
$100 \mathrm{mg} \mathrm{IZ}$ & 7 & $1.02 \pm 0.38$ & $0.96 \pm 0.42$ & $0.43 \pm 0.13$ & $0.84 \pm 0.25$ & $0.93 \pm 0.34$ \\
\hline
\end{tabular}

$\mathrm{IZ}=$ ischemic zone matrix placement, 3rd occlusion; $\mathrm{NIZ}=$ nonischemic zone matrix placement, 3rd occlusion; ${ }^{*} p>0.05$ for control vs. treated for all occlusions.

\section{TABLE 4}

Effects of epicardial D-sotalol on ventricular fibrillation

\begin{tabular}{|c|c|c|c|c|c|c|c|}
\hline \multirow[t]{2}{*}{ Group } & \multirow[t]{2}{*}{$N$} & \multicolumn{3}{|c|}{ Ist Occlusion (pre drug) } & \multicolumn{3}{|c|}{ 5th Occlusion ( $2 \mathrm{~h}$ of drug) } \\
\hline & & Ischemic VF & $\begin{array}{l}\text { Time to } \\
\text { ischemic VF } \\
\text { (min) }\end{array}$ & $\begin{array}{l}\text { Reperfusion } \\
\text { VF }\end{array}$ & Ischemic VF & $\begin{array}{l}\text { Time to } \\
\text { ischemic VF } \\
\text { (min) }\end{array}$ & Reperfusion \\
\hline $200 \mathrm{mg} \mathrm{IZ}$ & 7 & $4 / 7(57.1 \%)$ & $6.5 \pm 1.2$ & $3 / 3(100 \%)$ & $6 / 7(85.1 \%)$ & $6.2 \pm 1.1$ & $1 / 1(100 \%)$ \\
\hline $200 \mathrm{mg}$ NIZ & 7 & $4 / 7(57.1 \%)$ & $7.3 \pm 0.9$ & $3 / 3(100 \%)$ & $7 / 7(100 \%)$ & $5.9 \pm 0.6$ & $0 / 0(0 \%)$ \\
\hline $100 \mathrm{mg} \mathrm{IZ}$ & 7 & $6 / 7(85.7 \%)$ & $5.1 \pm 1.0$ & $1 / 1(100 \%)$ & $3 / 6(50 \%)$ & $6.8 \pm 1.2$ & $3 / 3(100 \%)$ \\
\hline Vehicle (control) & 9 & $7 / 9(77.7 \%)$ & $5.7 \pm 1.0$ & $2 / 2(100 \%)$ & $8 / 9(88.8 \%)$ & $5.4 \pm 0.8$ & $1 / 1(100 \%)$ \\
\hline
\end{tabular}

chemia. Isolated premature beats were significantly reduced in the $200 \mathrm{mg}$ matrix ischemic zone placement studies (Table 1). A similar trend, although not statistically significant was noted for couplets in the $200 \mathrm{mg}$ matrix ischemic zone results, and with both the $200 \mathrm{mg}$ matrix nonischemic zone studies and the $100 \mathrm{mg}$ sotalol matrices when placed in the ischemic zone (Table 2). However, none of the matrices were effective for significantly inhibiting isolated premature beats or couplets when placed in the nonischemic zone.

D-sotalol-MPU5 matrices were effective for inhibiting ventricular tachycardia during acute 

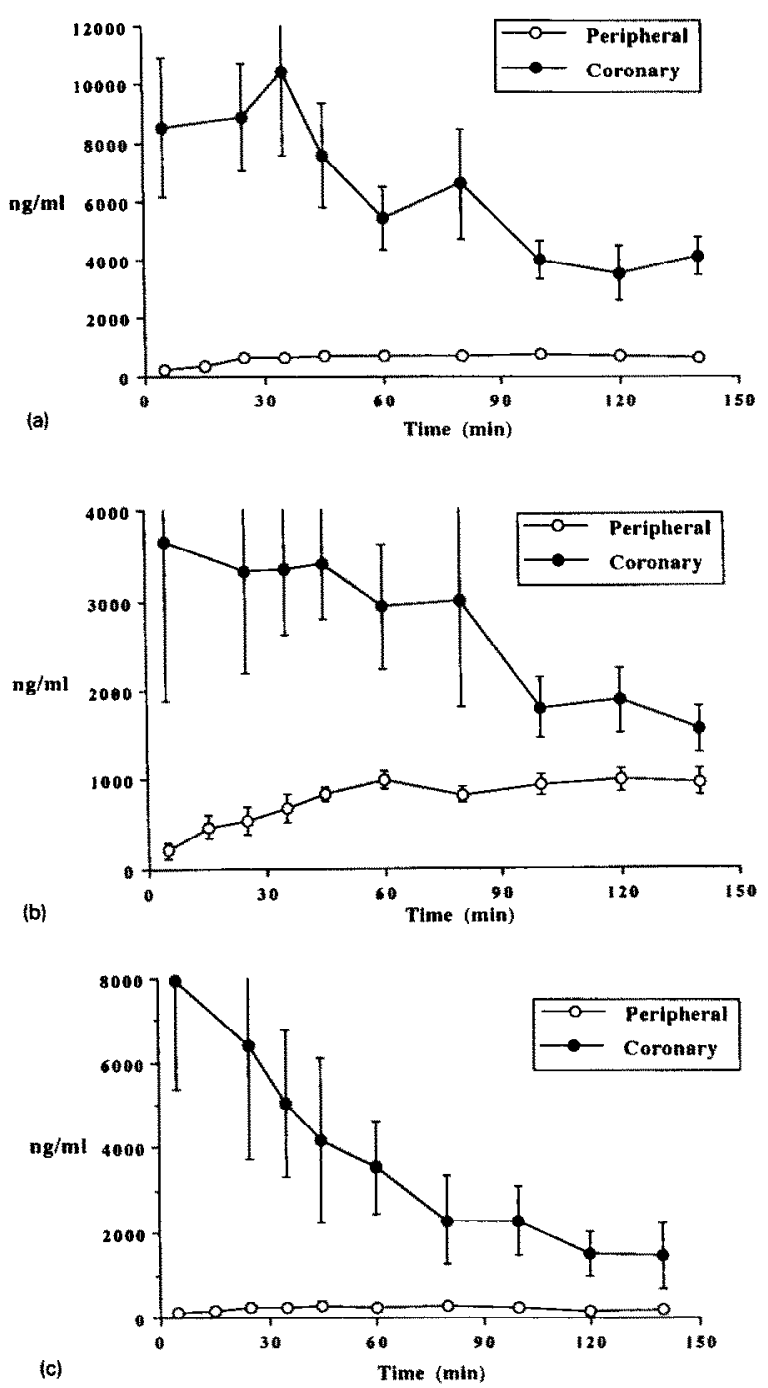

Fig. 2. Plasma sotalol levels comparing peripheral sotalol to coronary venous concentrations in animals $(N=7)$ receiving $200 \mathrm{mg}$ D-sotalol controlled release matrices placed prior to the 3 rd of 5, 10 min LAD occlusions (hourly), on the ischemic zone (A) of the left ventricular epicardium in open chest dogs, compared to $200 \mathrm{mg}$ matrix nonischmic zone studies (B), and $100 \mathrm{mg}$ matrix ischemic zone experiments

(C).

ischemia; however, these results were not statistically significant for any of the drug systems including the $200 \mathrm{mg}$ matrices placed in the ischemic zone (Table 1). As shown in Table 3, beginning with the third occlusion, there was a progressive decrease in the incidence of VT,

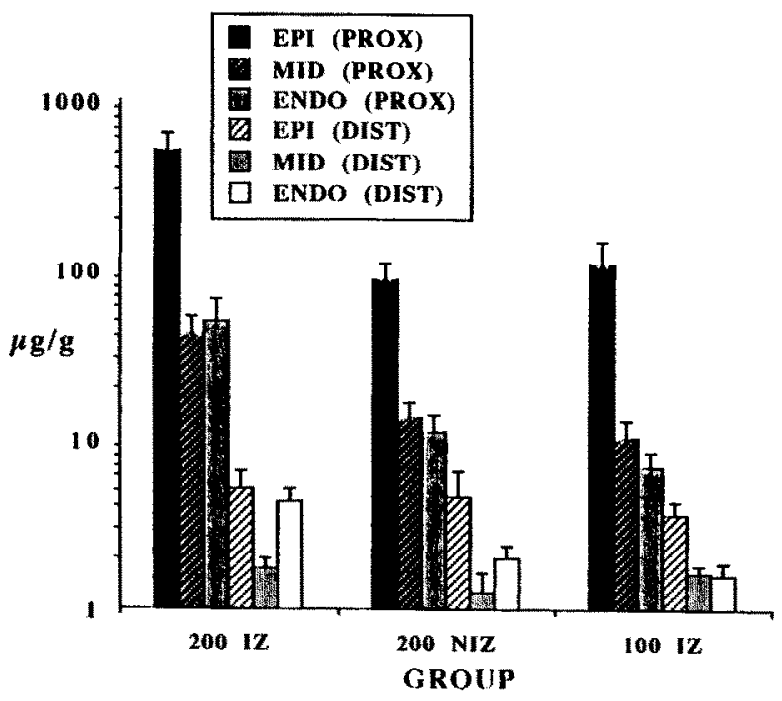

Fig. 3. Myocardial sotalol concentration in stratified samples, from animals receiving $200 \mathrm{mg}$ or $100 \mathrm{mg}$ controlled release matrices placed on either the left ventricular ischemic zone (IZ) or nonischemic zone (NIZ) (200 mg only), of the epicardium in animals undergoing repeated $10 \mathrm{~min}$ complete occlusions (hourly) of the left anterior descending coronary artery (LAD). Proximal (PROX) and distal (DIST) samples compared levels in myocardial strata including epicardium (EPI), midmyocardium (MID), and endocardium (END).

which declined to the lowest levels by the time of the fifth LAD occlusion for the $200 \mathrm{mg}$ ischemic zone matrix placement studies. When $200 \mathrm{mg}$ matrices were placed in the nonischemic zone, no trend in reducing VT was observed. Similarly, $100 \mathrm{mg}$ matrices placed in either the ischemic or nonischemic zones were also not significantly effective compared to controls (Table 3).

By the 5th LAD occlusion ventricular fibrillation occurred after $6.2 \pm 1.1 \mathrm{~min}$. in $85.7 \%$ of dogs treated with $200 \mathrm{mg} D$-sotalol controlled release matrices placed in the ischemic zone (Table 4); $200 \mathrm{mg}$ nonischemic zone matrices were similarly ineffective (Table 4). These data did not differ significantly from control. Ischemic zone placements of $100 \mathrm{mg}$ matrices were also ineffective for ventricular fibrillation (Table 4). Furthermore, reperfusion ventricular fibrillation occurred in all animals not fibrillating during acute ischemia, regardless of D-sotalol controlled re- 
lease therapy. Thus, controlled release D-sotalol therapy, although effective for ischemia-induced ventricular arrhythmias, was ineffective for VF induced by either ischemia or reperfusion.

Matrix retrieval analyses demonstrated that the $200 \mathrm{mg}$ D-sotalol ischemic zone matrices delivered a mean net dose of $1.2 \mathrm{mg} / \mathrm{kg}$. D-sotalol peripheral plasma levels in the $200 \mathrm{mg}$ ischemic zone studies ranged from $0.23 \mu \mathrm{g} / \mathrm{ml}$ to $0.78 \mu \mathrm{g}$ / $\mathrm{ml}$; however, simultaneous coronary venous levels were much higher, ranging from $3.53 \mu \mathrm{g} / \mathrm{ml}$ to $10.44 \mu \mathrm{g} / \mathrm{ml}$ (Fig. 2). Despite the lack of statistically significant efficacy for ventricular arrhythmias of the nonischemic zone matrices, or the $100 \mathrm{mg}$ matrices regardless of placement site, these controlled release administrations also resulted in comparable plasma levels to those noted with the efficacious $200 \mathrm{mg}$ ischemic zone matrices. For example, $200 \mathrm{mg}$ nonischemic zone Dsotalol matrix placement resulted in coronary venous levels ranging from $1.54 \mu \mathrm{g} / \mathrm{ml}$ to 3.64 $\mu \mathrm{g} / \mathrm{ml}$, with $0.20 \mu \mathrm{g} / \mathrm{ml}$ to $0.99 \mu \mathrm{g} / \mathrm{ml}$ peripherally by comparison (Fig. 2). Furthermore, 100 $\mathrm{mg}$ ischemic zone matrix implants resulted in coronary plasma levels ranging from $1.45 \mu \mathrm{g} / \mathrm{ml}$ to $7.95 \mu \mathrm{g} / \mathrm{ml}$ and peripheral plasma sotalol levels ranging from $0.13 \mu \mathrm{g} / \mathrm{ml}$ to $0.30 \mu \mathrm{g} / \mathrm{ml}$ (Fig. 2).

Myocardial sotalol levels in the $200 \mathrm{mg}$ ischemic zone studies were highest in the proximal epicardium, and next highest in the proximal midmyocardium and the endocardium; proximal mid- and endocardial sotalol levels were comparable (Fig. 3). However, distal sotalol levels in the $200 \mathrm{mg}$ ischemic zone studies were significantly lower than proximal endocardial levels, indicating a relatively rapid gradient distribution of D-sotalol to distal myocardial regions. Although $200 \mathrm{mg}$ nonischemic zone sotalol matrix placements demonstrated a proximal to distal drug distribution comparable to ischemic zone placement, total sotalol levels were lower in the nonischemic zone matrix studies compared to the ischemic zone studies (Fig. 3 ). Similarly, $100 \mathrm{mg}$ matrices placed in the ischemic zone showed a comparable drug distribution pattern to the $200 \mathrm{mg}$ matrices, but with lower sotalol tissue levels (Fig. 3 ).

\section{Discussion}

The present studies have demonstrated the class III antiarrhythmic agent, D-sotalol, incorporated into polyurethane controlled release matrices was effective for preventing ventricular arrhythmias induced by acute ischemia. The acute ischemia animal model [6] used employing repeated LAD occlusions mimics the pathophysiology of an acute coronary obstruction, with associated potentially fatal ventricular arrhythmias. Animal models of acute-ischemic ventricular arrhythmias $[6,15]$ have not been widely used due to difficulties in assessing the typically nonsustained ventricular ectopy observed. However, our prior studies with controlled release verapamil [6] established the reliability of the present model system for investigating this clinically relevant setting.

Among the determinants of controlled release D-sotalol efficacy were ischemic zone matrix placement, as well as the use of $200 \mathrm{mg}$ matrices, which provided coronary venous sotalol levels in the same range as therapeutic peripheral levels observed clinically [16-18]. Also of significance is the fact that peripheral plasma levels in the 200 $\mathrm{mg}$ ischemic zone studies were well below those known to be clinically effective [16-18], and this result implies that the pharmacologic effects of D-sotalol administered to the epicardium were achieved locally.

It is of interest, however, that despite significant differences in efficacy between $100 \mathrm{mg}$ and $200 \mathrm{mg}$ sotalol matrices depending upon the site of placement, comparable coronary and peripheral plasma levels were obtained in the ischemic zone placement studies for both the $100 \mathrm{mg}$ and $200 \mathrm{mg}$ matrices. Thus, although coronary plasma concentration was enhanced with epicardial controlled release, the absolute drug levels achieved did not predict efficacy in the ischemic zone studies. However, the $200 \mathrm{mg}$ nonischemic zone matrix placement results demonstrated coronary venous plasma levels which were almost half those observed in the ischemic zone experiments.

Myocardial concentrations may be a better in- 
dication of therapeutic effectiveness, since enhanced proximal myocardial sotalol levels were noted with $200 \mathrm{mg}$ ischemic zone matrix placement. It may be that the drug retention is favored in a myocardial region with an impaired regional circulation, such as the repeatedly ischemic myocardium in the present experiments. Research by others has demonstrated enhanced myocardial drug levels of lidocaine $[19,20]$ and propranolol $[21,22]$ in infarcted tissue compared to normally perfused myocardium. Thus, inadequate perfusion of a myocardial region may in fact favor drug retention, and therefore work to the advantage of direct cardiac administration of antiarrhythmic agents. Alternatively, since sotalol is a zwitterion with a relatively narrow $\mathrm{pH}$ range in which charge dependent uptake variability may arise, minute $\mathrm{pH}$ differences within the ischemic zone due to cellular damage $[23,24]$ may also serve to partition the drug within this region, inhibiting diffusion into the circulation.

It is further of interest that controlled release D-sotalol therapy in the present studies did not favorably affect the occurrence of ventricular fibrillation during either acute ischemia or reperfusion. Prior work by our group using the same animal model with the calcium channel blocker, verapamil, incorporated into control release matrices, demonstrated a significant reduction in VF occurring during acute ischemia, but not reperfusion [6]. It is noteworthy that D-sotalol in the present study, and verapamil in our previous work were effective for preventing ventricular arrhythmias associated with acute ischemia. However, verapamil effectively inhibited VT and VF regardless of the site of matrix placement, while sotalol only significantly inhibited isolated ventricular ectopy if matrices were placed in the ischemic zone. Other agents such as the class I agent, lidocaine [25], or the beta adrenergic blocker propranolol [26] were not significantly effective in this acute-ischemia model system. Thus, fundamental mechanistic differences in drug action may also determine specific arrhythmia outcomes, rather than simply achieving intensely high regional concentration of an antiar- rhythmic agent in a nonspecific fashion.

Although intravenous D-sotalol comparisons were not within the scope of the present research, the results of others have demonstrated that $D$-sotalol is an effective antiarrhythmic agent when administered intravenously. Nevertheless, the dosages ( 8 to $10 \mathrm{mg} / \mathrm{kg}$ ) used in these previous investigations $(7-10,16-18)$, and the resulting peripheral plasma levels (peak level, $50000 \mathrm{ng} / \mathrm{ml})(7-10,16-18)$, were significantly higher than those reported in the present study. However, the coronary venous D-sotalol levels in the present study were comparable to therapeutic levels reported by others (7-10, 16-19). Furthermore, Lynch et al, [27] demonstrated that in a multiple dose $(8 \mathrm{mg} / \mathrm{kg}$ intravenously every 8 hours) pretreatment regimen, D-sotolal suppressed the induction of ventricular tachycardia in an infarction model system, and also provided significant protection against the development of ventricular fibrillation in response to further acute ischemia in conscious post-infarction dogs. The net acute dose of D-sotalol in these studies, however was almost eight times greater than in the present studies. Furthermore, Lynch et al. [27] also demonstrated that the cumulative survival rates of multiple dose D-sotalol administration gave enhanced protection which was superior to single dose pretreatment. They hypothesized that the enhanced efficacy of the multiple dose regimen was the result of optimization of drug plasma concentrations or cardiac tissue levels or both in the post infarction dogs.

Furthermore, Brachmann et al. [28] have shown that the D-sotalol intravenous therapy resulted in an increased ventricular effective refractory period, and that this occurred to a greater extent in the infarct zone than in the normally perfused regions. The D-isomer of sotalol, also used in the present studies, was shown by this group [28] to be an effective antiarrhythmic and antifibrillatory agent in infarcted dogs previously subjected to a permanent complete occlusion of the LAD. Thus, prior work by others has demonstrated efficacy of D-sotalol for ventricular arrhythmias, although with suboptimal administration regimens compared to epicardial drug delivery. 
The matrices used in the present studies were comparable to polyurethane controlled release matrices utilized in previous arrhythmia research by our group [1-6]. The rapid early drug release characteristics of these matrices favors obtaining rapidly effective levels of the agents of choice, which would be most desirable for preventing or treating acute arrhythmia activity. This could be most clinically relevant in an acute setting such as early in the clinical course of a myocardial infarction (with a catheter-matrix placement strategy), or postcardiac surgery (with epicardial matrices placed at closure).

It is of further interest, that in vivo matrix release studies using the D-sotalol matrices in the present work, demonstrated a release pattern that was similar to that noted in vitro, but with a release rate that was roughly $50 \%$ of that noted in vitro. This has been observed previously by our group [5] in studies of lidocaine release from epicardial matrices, and is most likely due to the fact that in vivo, drug release occurs only at the epicardial contacting surface, rather than from all surfaces of the matrix as would take place with in vitro incubations.

Controlled release matrices may be formulated with release profiles that can be extended for decades, as has been demonstrated using controlled release systems for prevention of cardiovascular calcification in animal model experiments of bioprosthetic heart valve calcification $[29,30]$. In addition, multiyear controlled release is currently in clinical use with a steroid eluting cardiac pacing lead [31 ]. In this drug delivery system, the electrode tip contains a silicone rubber-dexamethasone drug delivery system which slowly releases dexamethasone into the implant site of a pacemaker electrode [27]. This effectively prevents acute inflammatory reaction and chronic scar tissue buildup, which would otherwise adversely affect electrical pacing capture thresholds. Thus, for the prevention of ventricular arrhythmias in a long term setting, extended controlled release implants may be readily formulated and tailored to suit clinical needs.

In conclusion, D-sotalol controlled release ma- trices used as epicardial implants in dogs undergoing repeated LAD occlusion were effective for preventing ischemia-induced ventricular tachycardia, couplets, and isolated premature beats. However, epicardial sotalol was only effective when $200 \mathrm{mg}$ matrices were used as ischemic zone implants. In addition, these matrices were not effective for preventing ischemia or reperfusion induced ventricular fibrillation. Dsotalol controlled release resulted in enhanced coronary plasma drug concentration compared to simultaneous peripheral plasma drug levels. Myocardial drug concentrations were most enhanced in the ischemic myocardium, although a relatively rapid distribution of D-sotalol to distal sites was also observed.

\section{Acknowledgments}

The authors thank Mrs. Catherine Wongstrom and Ms. Jill Van Cise for preparing the manuscript. This work was supported in part by NHLBI Grant HL41663, and a Grant-in-Aid from the American Heart Association (890654). Dr. Levy's efforts also supported by an American Heart Association Established Investigatorship (86-0166).

\section{References}

1 A. Sintov, W. Scott, M. Dick and R.J. Levy, Cardiac controlled release for arrhythmia therapy: lidocaine-polyurethane matrix studies, J. Controlled Release 8 (1988) 157-165.

2 A. Sintov, W.A. Scott, K.P. Gallagher and R.J. Levy, Conversion of ouabain-induced ventricular tachycardia in dogs with epicardial lidocaine: pharmacodynamics and functional effects, Pharmaceut. Res. 7 (1990) 2833.

3 A. Sintov, W. Scott, R. Siden and R.J. Levy, Polymer matrices for delivery of lidocaine to the myocardium, in: W.D. Ensminger and J.L. Selam (Ed), Update in Drug Delivery Systems, Futura Publishing Co, Mount Kisco, NY, 1989, pp. 325-332.

4 A. Sintov, R. Siden and R.J. Levy, Sensitive high-performance liquid chromatographic assay using 9-fluoroenylmethylchloroformate for monitoring controlledrelease lidocaine in plasma, J. Chromatog. Biomed. Appl. 496 (1989) 335-344. 
5 A. Sintov, W.A. Scott, R. Siden and R.J. Levy, Efficacy of epicardial controlled-release lidocaine for ventricular tachycardia induced by rapid ventricular pacing in dogs, J. Cardiovasc. Pharm. 16 (1990) 812-817.

6 R. Siden, A. Kadish, W. Flowers, L. Kutas, B.K. Bieneman, J. DePietro, J.P. Jenkins, K.P. Gallagher and R.J. Levy, Epicardial controlled release verapamil prevents ventricular tachycardia episodes induced by acute ischemia in a canine model, J. Cardiovasc. Pharm., in press.

7 M.N. Hicks and S.M. Cobbe, Attenuation of the rise in extracellular potassium concentration during myocardial ischemia by DL-sotalol and D-sotalol, Cardiovasc. Res. 24 (1990) 404-410.

8 A.W. Gomoll, R.F. Lekich, M.J. Bartek, C.R. Comereski and M.J. Antonaccio, Comparability of the electrophysiologic responses and plasma and myocardial tissue concentrations of sotalol and its D-stereoisomer in the dog, J. Cardiovasc. Pharmacol. 16 (1990) 204-211.

9 H. Bjornstad, P.M. Tande and H. Refsum, Class III antiarrhythmic action of D-sotalol during hypothermia, Am. Heart. J. 121 (1991) 1429-1436.

10 C. Funk-Brentano, D.J. Silbertein, D.M. Roden, A.J. Wood and R.L. Woosley, A mechanism of D-sotalol effects on heart rate not related to beta-adrenoceptor antagonism, Br. J. Clin. Pharmacol. 30 (1990) 195-202.

11 M. Pagani, S.F. Vatner, H. Baig and E. Braunwald, Initial myocardial adjustments to brief period of ischemia and reperfusion in the conscious dog, Cir. Res. 43 (1978) 83-92.

12 J.M. Poirier, P. Jaillon and G. Cheymol, Quantitative liquid chromatographic determination of sotalol in human plasma, Therap. Drug. Monit. 8 (1986) 474-477.

13 J.M. Poirier, M. Lebot and G. Cheymol, Rapid and sensitive column liquid chromatographic determination of sotalol in plasma, J. Chromatogr. 493 (1989) 409-413.

14 M.A. Lefebvre, J. Girault, M.C. Saux and J.B. Fourtillan, Fluoremetric high-performance liquid chromatographic determination of sotalol in biological fluids, $\mathrm{J}$. Pharm. Sci. 69 (1980) 1216-1217.

15 V. Elharrar, W.E. Gakum and D.P. Zipes, Effects of drugs on conduction delay and incidence of ventricular arrhythmias induced by acute coronary occlusion in dogs, Am. J. Cardiol. 39 (1977) 544-549.

16 M.J. Antonaccio and A. Gomoll, Pharmacology, pharmacodynamics, and pharmacokinetics of sotalol, Am. J. Cardiol. 65 (1990) 12A-21A.

17 B.N. Singh, P. Deedwania, K. Nademanee, A. Ward and E.M. Sorkin, Sotalol. A review of its pharmacodynamic and pharmacokinetic properties, and therapeutic use, Drugs 34 (1987) 311-349.

18 M. Dumas, P. D'Athis, J.F. Besancenot, V. ChadointNoudeau, J.M. Chalopin, G. Rifle and A. Escousse, Variations of sotalol kinetics in renal insufficiency, Int. J. Clin. Pharmacol. Ther. Toxicol. 27 (1989) 486-489.

19 R.A. Zito, V.J. Caride, T. Holford and B.L. Zaret BL, Regional myocardial lidocaine concentration following continuous intravenous infusion early and later after myocardial infarction, Am. J. Cardiol. 50 (1982) 497502.

20 M.S. Chow, R.A. Ronfeld, R.A. Hamilton, R. Helmink and A. Fieldman, Effect of external cardiopulmonary resuscitation on lidocaine pharmacokinetics in dogs, $J$. Pharmacol. Exp. Ther. 224 (1983) 531-537.

21 R.E. Kates and P. Jaillon, A model to describe myocardial drug disposition in the dog, J. Pharmacol. Exp. Ther. 214 (1980) 31-36.

22 G.W. Hamilton, K.A. Narahara, H. Yee, J.L. Ritchie, D.L. Williams and K.L. Gould, Myocardial imaging with thallium-201: effect of cardiac drugs on myocardial images and absolute tissue distribution, J. Nucl. Med. 19 (1978) 10-16.

23 Y. Hara, K. Ichihara and Y. Abiko, MCI-176, a novel calcium blocker, attentuates the ischemic myocardial acidosis induced by coronary artery occlusion in dogs, J. Pharmacol. Exp. Ther. 245 (1988) 305-310.

24 K. Ichihara, M. Ichihara and Y. Abiko, Involvement of beta adrenergic receptors in decrease of myocardial $\mathrm{pH}$ during ischemia, J. Pharmacol. Exp. Ther. 209 (1979) 275-281.

25 R. J. Levy, S. F. Bolling, R. Siden, A. Kadish, Y. Pathak, P. Dorostkar, A. Sintov, G. Golomb and T.P. Johnston, Polymeric controlled release of cardiovascular drugs, in: C. Gebelein (Ed.), Cosmetic and Pharmaceutical Applications of Polymers, Plenum Press, New York, 1992, pp. 231-238.

26 R. Siden, W.E. Flowers and R.J. Levy, Epicardial propranolol for ventricular arrhythmias in dogs: matrix formulation and characterization, Biomaterials (1992) in press.

27 J.J. Lynch, L.A. Coskey, D.G. Montgomery and B.R. Lucchesi, Prevention of ventricular fibrillation by dextrorotatory sotalol in a conscious canine model of sudden coronary death, Am. Heart. J. 109 (1985) 949-958.

28 J. Brachmann, J. Senger, I. Aidonidis, I. Rizos and W. Kuber, Antiarrhythmic Class III effects of D-sotalol in conscious dogs with subacute myocardial infarction, $J$. Am. Coll. Cardiol. 5 (1985) 466-471.

29 G. Golomb, M. Dixon, M.S. Smith, F.J. Schoen and R.J. Levy, Controlled release drug delivery of diphosphon. ate to inhibit bioprosthetic heart valve calcification: release rate modulation with silicone matrices via drug solubility and membrane coating, J. Pharm. Sci. 76 (1988) 271-276.

30 T.P. Johnston, F.J. Schoen and R.J. Levy, Prevention of calcification of bioprosthetic heart valve leaflets by $\mathrm{Ca}^{2+}$ diphosphonate pretreatment, J. Pharm. Sci. 77 (1988) 740-744.

31 H. Mond, K. Stokes, J. Helland, L. Grigg, P. Kertes, B. Pate and D. Hunt, The porous titanium steroid eluting electrode: a double blind study assessing the stimulation threshold effects of steroid, PACE 11 (1988) 214-219. 